13.4

\title{
Генерация импульсов сверхизлучения терагерцового диапазона в процессе вынужденного рассеяния лазерного излучения на попутном сильноточном релятивистском электронном пучке
}

\author{
(C) Н.С. Гинзбург, Л.А. Юровский, А.В. Назаровский, А.С. Сергеев, И.В. Зотова
}

Институт прикладной физики РАН, Нижний Новгород, Россия

E-mail: ginzburg@appl.sci-nnov.ru

Поступило в Редакцию 22 июля 2020г.

В окончательной редакции 5 августа 2020г.

Принято к публикации 6 августа 2020 г.

\begin{abstract}
Анализируется возможность генерации импульсов сверхизлучения терагерцового диапазона при попутном рассеянии лазерного импульса на сильноточном релятивистском электронном пучке с преобразованием частоты вниз. Рассеянное излучение распространяется во встречном (по отношению к поступательной скорости частиц) направлении. Показано, что при использовании в качестве накачки импульсов с энергией $3 \mathrm{~kJ}$ на длине волны $\sim 1 \mu \mathrm{m}$ при оптимизации длительности лазерного импульса и длины области взаимодействия возможна генерация импульсов рассеянного встречного (по отношению к поступательной скорости частиц) излучения с мощностью порядка $580 \mathrm{~kW}$ и длительностью $30 \mathrm{ps}$ на длине волны $100 \mu \mathrm{m}$.
\end{abstract}

Ключевые слова: терагерцовые импульсы, вынужденное рассеяние.

DOI: 10.21883/PJTF.2020.23.50339.18479

Прогресс в создании мощных импульсных лазеров с высоким энергозапасом (более $1 \mathrm{~kJ}$ ) наряду с основными приложениями, направленными на инициацию управляемого термоядерного синтеза, делает актуальным анализ альтернативных приложений таких лазеров. В частности, в работе [1] показана возможность использования многолучевых комплексов для создания некогерентной накачки в лазерах на свободных электронах, основанных на вынужденном комптоновском рассеянии волн, когда поле накачки распространяется навстречу релятивистскому электронному пучку, а рассеянное излучение - в попутном направлении. В таких условиях доплеровского преобразования частоты вверх обеспечивается генерация частично когерентного излучения рентгеновского диапазона. В настоящей работе анализируется альтернативная схема, при которой волна накачки попутна электронному пучку, а рассеянное излучение распространяется во встречном направлении и имеет место преобразование частоты вниз. При оптической частоте накачки и умеренных энергиях электронов порядка нескольких $\mathrm{MeV}$ частота рассеянного излучения будет находиться в терагерцовом (THz) диапазоне.

Таким образом, исследуемая схема может рассматриваться как разновидность схем генерации THz-излучения при воздействии лазерного излучения на нелинейную среду, в качестве которой в данном случае предлагается использовать электронный пучок. При этом в отличие от исследованных ранее механизмов [2,3] генерируемое излучение будет представлять собой не моноимпульс, а импульс с высокочастотным заполнением, включающий до $10-10^{2}$ периодов колебаний THz-диапазона.

С другой стороны, по направлению распространения сигнала относительно электронного пучка процесс ге- нерации THz-импульсов в пренебрежении истощением волны накачки в значительной степени аналогичен процессу генерации импульсов сверхизлучения (СИ) при взаимодействии электронов со встречной сигнальной волной в периодической замедляющей системе типа лампы обратной волны [4]. Эффективность такого процесса для генерации мощных микроволновых импульсов неоднократно подтверждена экспериментально $[5,6]$. В исследуемом случае вынужденного рассеяния возможность синхронного взаимодействия электронов со встречной волной обеспечивается за счет осцилляций, приобретаемых электронами в поле волны накачки. Связь между частотами и волновыми числами попутной волны накачки (индекс $i$ ) и встречной рассеянной сигнальной волны (индекс $s$ ) имеет вид

$$
\omega_{i}-k_{i} V_{\|}=\omega_{s}+k_{s} V_{\|},
$$

где $V_{\|}-$поступательная скорость электронов. Это условие может рассматриваться как условие синхронизма между электронами и виртуальной комбинационной волной $V_{\|}=V_{c}=\omega_{c} / k_{c}$ с частотой $\omega_{c}=\omega_{i}-\omega_{s}$ и волновым числом $k_{c}=k_{i}+k_{s}$.

С учетом импульсного характера полей, представляя поля накачки и рассеянного излучения в виде

$$
\mathbf{A}_{j}=\operatorname{Re}\left[\mathbf{y}_{0} \hat{A}_{j}(z, t) \exp \left(i \omega_{j} t-i k_{j} z\right)\right], \quad j=i, s
$$

(где $\hat{A}_{j}(z, t)$ - медленно меняющиеся амплитуды волн), процесс рассеяния в условиях синхронизма (1) можно описать следующей системой уравнений [7]:

$$
\left(\frac{1}{V_{g r, i}} \frac{\partial}{\partial t}+\frac{\partial}{\partial z}\right) \alpha_{i}=i \frac{\omega_{p \perp}^{2}}{4 \omega_{i} c} \rho_{1} \alpha_{s}
$$




$$
\begin{gathered}
\left(\frac{1}{V_{g r, s}} \frac{\partial}{\partial t}-\frac{\partial}{\partial z}\right) \alpha_{s}=i \frac{\omega_{p \perp}^{2}}{4 \omega_{s} c} \alpha_{i} \rho_{1}^{*} \\
\left(\frac{1}{V_{\|}} \frac{\partial}{\partial t}+\frac{\partial}{\partial z}\right)^{2} \theta=\left(\frac{\omega_{c}}{c}\right)^{2} \operatorname{Im}\left[\mu \alpha_{i} \alpha_{s}^{*} e^{i \theta}+\frac{\omega_{p \|}^{2}}{\omega_{c}^{2}} \rho_{1} e^{i \theta}\right]
\end{gathered}
$$

Здесь $\alpha_{j}=e \hat{A}_{j} / \sqrt{2} \gamma_{0} m_{e} c^{2}-$ нормированные амплитуды сигнала и накачки, $\rho_{1}=\frac{1}{\pi} \int_{0}^{2 \pi} e^{-i \theta} d \theta_{0}-$ амплитуда первой гармоники модуляции плотности пучка в поле комбинационной волны, $\theta=\omega_{c} t-k_{c} z-$ фаза электронов относительно комбинационной волны, $\mu=\gamma_{0}^{-2}-$ параметр группировки, $\omega_{p \|}=\omega_{p \perp} / \gamma_{0}$ и $\omega_{p \perp}=\sqrt{4 \pi e \rho_{0} / \gamma_{0} m_{e}}-$ продольная и поперечная плазменные частоты, $\rho_{0}-$ невозмущенная плотность пучка, $e$ и $m_{e}-$ заряд и масса покоя электрона.

Пренебрегая отличием групповой скорости попутной волны накачки от поступательной скорости электронов и переходя к нормированным безразмерным переменным и параметрам, приведем систему уравнений (3)-(5) к виду

$$
\begin{gathered}
\frac{\partial}{\partial Z} a_{i}=i G \rho_{1} a_{s}, \\
\left(\frac{\partial}{\partial \tau}-\frac{\partial}{\partial Z}\right) a_{s}=i a_{i} \rho_{1}^{*}, \\
\frac{\partial^{2} \theta}{\partial Z^{2}}=\operatorname{Im}\left[a_{i} a_{s}^{*} e^{i \theta}+q^{2} \rho_{1} e^{i \theta}\right],
\end{gathered}
$$

где $Z=z C \omega_{c} / c, \quad \tau=\omega_{c} C(t-z / c) / 2$-,запаздывающее“" время, $a_{i}=\alpha_{i} / \alpha_{i 0}$,

$$
\begin{gathered}
a_{s}=\mu \alpha_{s} \alpha_{i 0}^{*} C^{-2} \\
G=\left(\left(\frac{I_{e}}{17[\mathrm{kA}]}\right)^{4}\left(\frac{8.7[\mathrm{GW}]}{P_{i 0}}\right)^{2} \frac{\pi^{2}}{S^{2}} \frac{\omega_{i}}{\omega_{s}} \frac{\gamma_{0}^{4} c^{4}}{16 \omega_{c}^{4}}\right)^{1 / 3}
\end{gathered}
$$

— коэффициент истощения накачки,

$$
C=\left(\frac{I_{e}}{17[\mathrm{kA}]} \frac{P_{i 0}}{8.7[\mathrm{GW}]} \frac{4 \pi^{2} c^{4} \gamma_{0}^{-5}}{S^{2} \omega_{i}^{2} \omega_{c} \omega_{s}}\right)^{1 / 3}
$$

- параметр усиления (параметр Пирса), $P_{i 0}$ - пиковое значение мощности импульса накачки, $I_{e}-$ полный ток, $S$ - площадь поперечного сечения электронного и волновых пучков, $q=\omega_{p \|} /\left(C \omega_{c}\right)$ - параметр пространственного заряда.

Пусть импульс накачки инжектируется с катодного конца: $\left.\alpha_{i}(\tau)\right|_{Z=0}=\alpha_{i 0} \sin ^{2}\left(\pi \tau / T_{i}\right)$, где $T_{i}=\omega_{c} C t_{i} / 2-$ нормированная длительность импульса накачки. В предположении, что отсутствует поле на частоте рассеянного излучения, падающее на систему извне $\left(\left.\alpha_{s}\right|_{Z=L}=0\right)$, процесс рассеяния в сверхизлучательном режиме развивается от малых начальных флуктуаций плотности электронного пучка, задаваемых параметром $r \ll 1$ :

$$
\left.\frac{\partial \theta}{\partial Z}\right|_{Z=0}=0,\left.\quad \theta\right|_{Z=0}=\theta_{0}+r \cos \theta_{0}, \quad \theta_{0} \in(0,2 \pi) .
$$

Проведем анализ процесса генерации THz-импульсов при параметрах реально существующих экспериментальных установок. В качестве накачки возьмем лазерный импульс с полной энергией $3 \mathrm{~kJ}$ и длиной волны $\lambda_{i}=1.053 \mu \mathrm{m}$, генерируемый одним из каналов лазерной установки „Луч“/,Искра-5“. Будем предполагать возможность изменения длительности лазерного импульса при сохранении полной энергии, а также возможность фокусировки волнового пучка до необходимых поперечных размеров [8-10]. Параметры электронного пучка, на котором происходит рассеяние импульса накачки, выберем близкими к параметрам пучков, формируемых ускорителем Scorpius (Лос-Аламос) [11,12]: ток пучка $I_{e}=2 \mathrm{kA}$, энергия частиц $2 \mathrm{MeV}$. Площадь поперечного сечения с учетом возможностей магнитной фокусировки $S=0.01 \mathrm{~cm}^{2}$. Площадь поперечного сечения импульса накачки будем считать равной соответствующему сечению электронного пучка.

Как следует из соотношений Мэнли-Роу и закона сохранения энергии, в исследуемой конфигурации генерируемый низкочастотный импульс СИ черпает энергию из высокочастотной волны накачки. При этом часть энергии поля накачки передается электронному пучку, т.е. электроны в среднем ускоряются. Тем не менее при выбранных физических параметрах коэффициент истощения накачки мал: $G \sim 10^{-8}$, и эффектом истощения накачки можно пренебречь. Заметим, что параметр пространственного заряда также относительно мал $\left(q^{2} \sim 0.02-0.1\right)$ и влияние эффектов кулоновского расталкивания незначительно. В таких предположениях процесс генерации импульсов рассеянного излучения характеризуется двумя параметрами: длиной пространства взаимодействия $L$ и длительностью импульса накачки $T_{i}$.

Моделирование процесса генерации импульсов СИ на основе системы уравнений (6) показало, что импульс накачки не меняет свою интенсивность и форму при проходе через пространство взаимодействия (рис. $1, a$ ). При этом комбинационное воздействие поля накачки и генерируемого импульса рассеянного излучения приводит к группировке электронов (рис. $1, b)$ и усилению импульса рассеянного излучения (рис. $1, c$ ), распространяющегося навстречу направлению движения электронов. Последний отбирает энергию у импульса накачки за счет взаимодействия с электронами в условиях синхронизма (1). Как видно из рис. $1, c$, максимальный темп роста амплитуды импульса СИ происходит в области наибольшей группировки электронов и замедляется при приближении к катодному концу пространства взаимодействия, что обусловлено малостью амплитуды модуляции плотности электронного потока в этой области (рис. $1, b)$.

На рис. 2, $a$ показана зависимость пиковой амплитуды импульсов рассеянного излучения от длины области рассеяния $L$ при различных нормированных длительностях импульса накачки $T_{i}$ и достаточно малой величине начальных флуктуаций плотности электронного пучка $r=0.001$. Видно, что существует оптимальное значение 


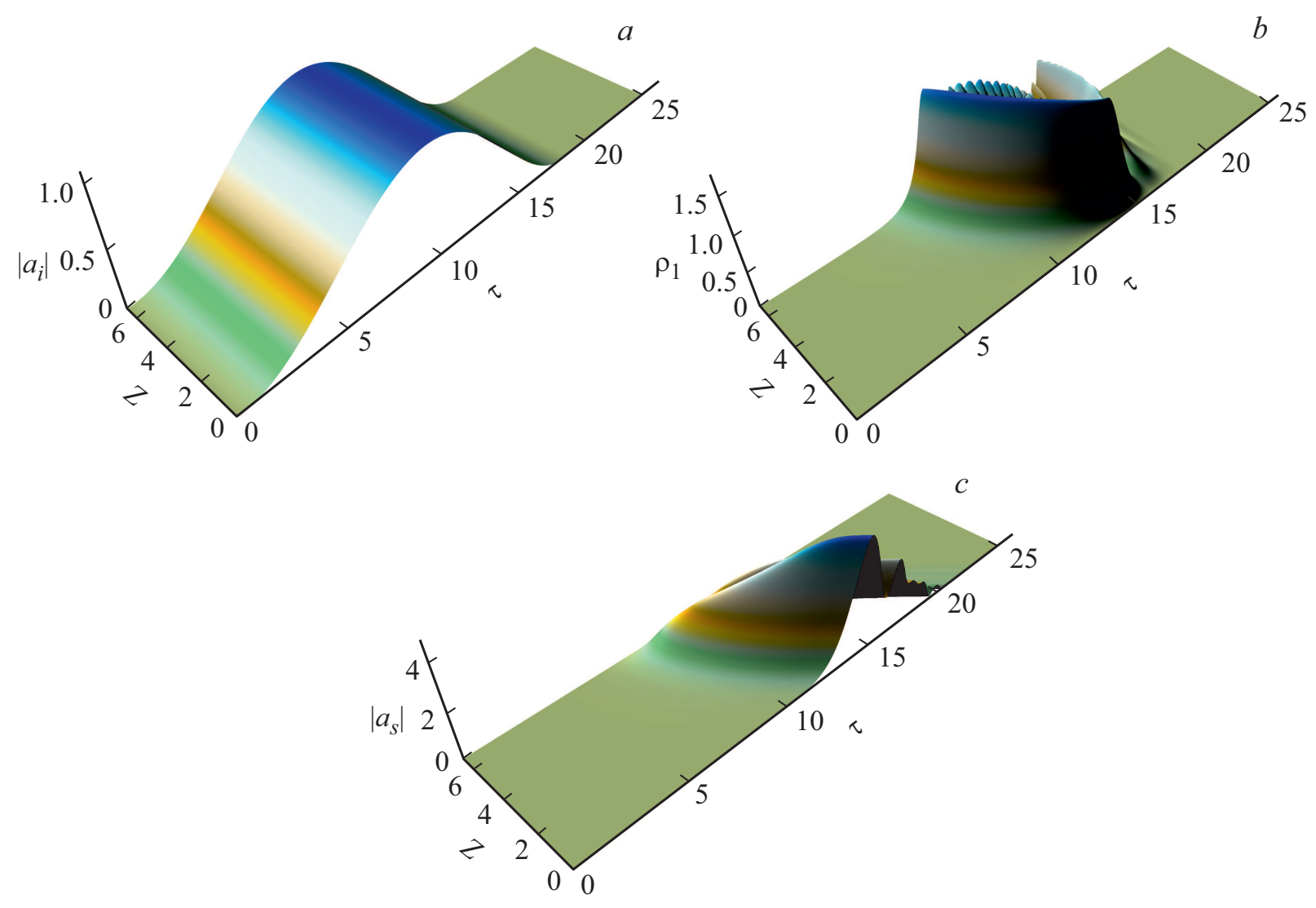

Рис. 1. Пространственно-временна́я эволюция импульса накачки $(a)$, амплитуды первой гармоники модуляции плотности пучка (b) и амплитуды рассеянного импульса $(c)$ при $T_{i}=18.5, L=6.7, r=0.001$.
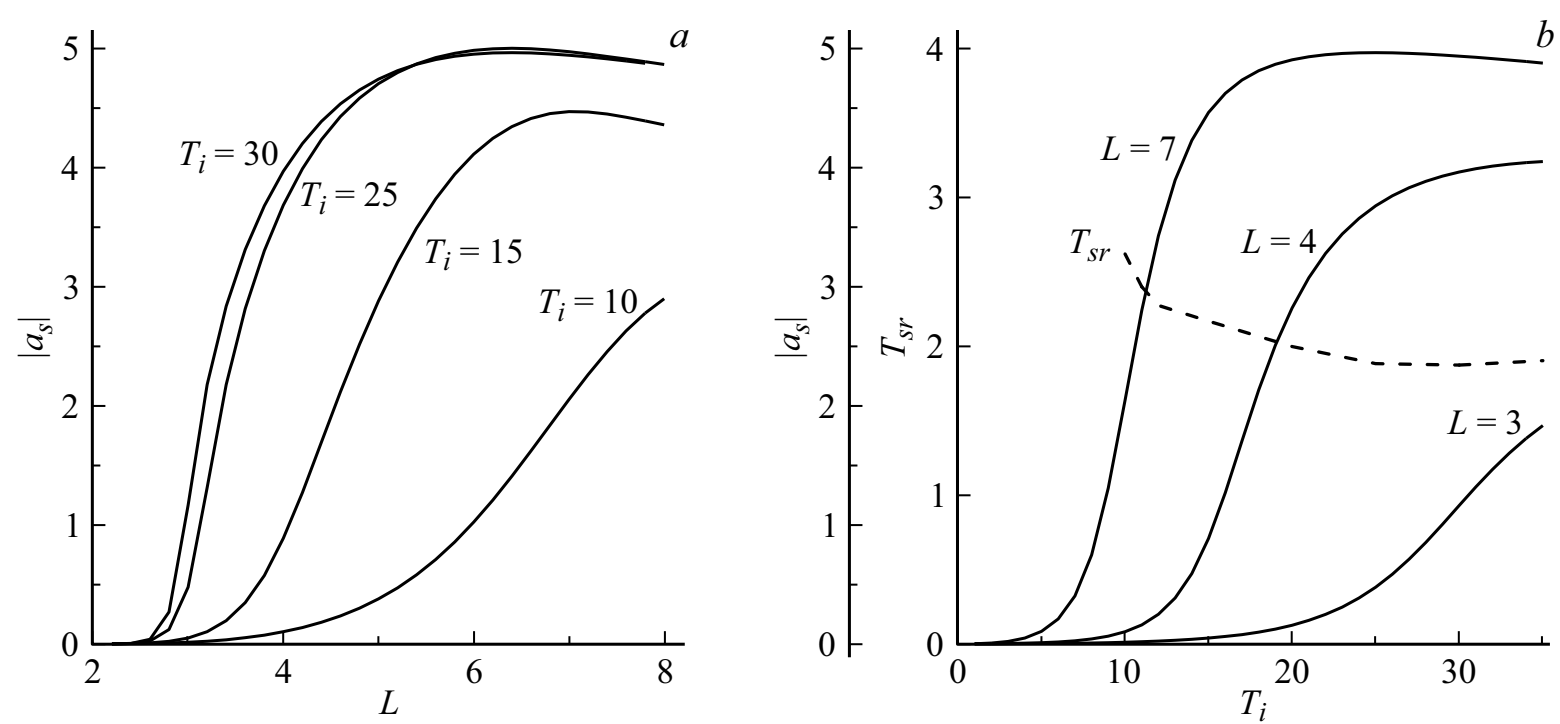

Рис. 2. Зависимости пиковой амплитуды импульса рассеянного излучения от длины пространства взаимодействия при различных значениях длительности импульса накачки $T_{i}(a)$ и от длительности импульса накачки при различных значениях длины области взаимодействия $L(b)$. На части $b$ приведена также зависимость нормированной длительности рассеянного импульса сверхизлучения $T_{s r}$ (штриховая кривая) от длительности импульса накачки при $L=7$.

длины $L \sim 6.5$. На рис. $2, b$ приведены зависимости пиковой амплитуды рассеянных импульсов от длительности импульса накачки $T_{i}$ при различных длинах области рассеяния. Здесь штриховой линией для указанной выше оптимальной длины показана длительность рассеянного импульса по полувысоте. 


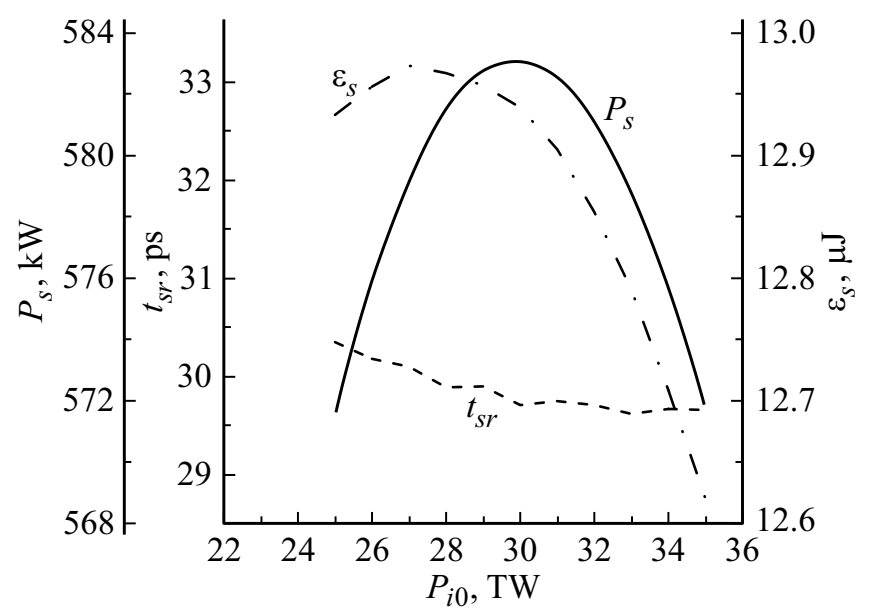

Рис. 3. Зависимости пиковой мощности (сплошная кривая), длительности (штриховая кривая) и полной энергии (штрихпунктирная кривая) рассеянного THz-импульса от мощности накачки при фиксированной его энергии $3 \mathrm{~kJ}$ и оптимальном значении длины пространства взаимодействия $l=15 \mathrm{~mm}$.

На основании проведенного моделирования можно найти зависимость параметров генерируемых THz-импульсов от интенсивности импульса накачки при его фиксированной энергии $3 \mathrm{~kJ}$. Предполагается, что длина области рассеяния достаточно велика $l=15 \mathrm{~mm}$, что соответствует нормированной длине $L \sim 6.5$. Очевидно, излишнее увеличение интенсивности импульса накачки при фиксированной энергии ведет к уменьшению его длительности, и соответственно процесс генерации импульсов рассеянного излучения может не успевать развиваться (рис. 2, $b$ ). Напротив, избыточное увеличение длительности импульса накачки приводит к тому, что сгенерированный THz-импульс, бегущий навстречу накачке, начинает взаимодействовать с ней в области малых напряженностей полей $\alpha_{i} \ll \alpha_{i 0}$. Следовательно, существует оптимальное значение длительности накачки, при котором отбор энергии из накачки происходит в области ее максимальной интенсивности $\alpha_{i} \sim \alpha_{i 0}$ (рис. 1), что обеспечивает максимальное значение пиковой мощности рассеянного THz-импульса (рис. 3). При этом, несмотря на то что при изменении длительности накачки нормированная длительность импульса СИ также претерпевает изменения (рис. $2, b$ ), реальная физическая длительность $t_{s r}$ остается неизменной (рис. 3). Таким образом, для энергии импульса накачки $3 \mathrm{~kJ}$ оптимальной является мощность накачки $P_{i 0}=30 \mathrm{TW}$ при длительности по полувысоте $0.1 \mathrm{~ns}$, которая позволяет генерировать импульсы рассеянного излучения на длине волны $100 \mu \mathrm{m}$ мощностью до $580 \mathrm{~kW}$ и энергией $12 \mu \mathrm{J}$ при длине области взаимодействия $15 \mathrm{~mm}$.

В заключение следует отметить, что оценки параметров THz-импульсов, генерируемых на основе исследованного в работе механизма, сопоставимы с параметрами импульсов, генерируемых в лазерах на свободных электронах THz-диапазона, основанных на излучении высокоэнергичных электронных сгустков в ондуляторных полях [13]. Следовательно, такой механизм может рассматриваться как перспективная возможность использования лазерного излучения существующих комплексов типа „Искра-5“ и им подобных для генерации перестраиваемых по частоте импульсов THz-диапазона. В качестве возможного развития предлагаемого механизма представляет интерес анализ системы, в которой процесс рассеяния происходит внутри резонатора, обеспечивающего многократное взаимодействие между импульсом накачки и сигнала и позволяющего увеличить суммарную энергию, отбираемую рассеянным импульсом у накачки.

\section{Финансирование работы}

Работа выполнена при поддержке Российского фонда фундаментальных исследований (проект № 19-3890059), а также частично в рамках госзадания (номер темы 0035-2019-0001).

\section{Конфликт интересов}

Авторы заявляют, что у них нет конфликта интересов.

\section{Список литературы}

[1] Гинзбург Н.С., Кочаровская Е.Р., Вилков М.Н., Сергеев А.С. // Письма в ЖТФ. 2018. Т. 44. В. 14. С. 3-12.

[2] Vicario C., Jazbinsek M., Ovchinnikov A.V., Chefonov O.V., Ashitkov S.I., Agranat M.B., Hauri C.P. // Opt. Express. 2015. V. 23. N 4. P. 4573-4580.

[3] Kartner F.X., Huang S., Granados E., Huang W.R., Hong K., Zapata L.E. // Opt. Lett. 2013. V. 38. N 5. P. 796-798.

[4] Гинзбург Н.С., Новожсилова Ю.В., Сергеев А.С. // Письма в ЖТФ. 1996. Т. 22. В. 9. С. 39-44.

[5] Korovin S.D., Eltchaninov .A., Rostov V.V., Shpak V.G., Yalandin M.I., Ginzburg N.S., Sergeev A.S., Zotova I.V. // Phys. Rev. E. 2006. V. 74. N 1. P. 016501 (1-8).

[6] Rostov V.V., Romanchenko I.V., Pedos M.S., Rukin S.N., Sharypov K.A., Shpak V.G., Shunailov S.A., Ul'masculov M.R., Yalandin M.I. // Phys. Plasmas. 2016. V. 23. N 9. P. 093103 (1-4).

[7] Братман В.Л., Гинзбург Н.С., Петелин М.И. // ЖЭТФ. 1979. T. 76. B. 3. C. $930-936$.

[8] Kirillov G.A., Murugov V.M., Punin V.T., Shemyakin V.I. // Laser Particle Beams. 1990. V. 8. N 4. P. 827-831.

[9] Анненков В.И., Багрецов В.А., Безуглов В.Г., Виноградский Л.М., Гайдаш В.А., Галахов И.В., Гашеев А.С., Гузов И.П., Задорожный В.И., Ерошенко В.А., Ильин А.Ю., Каргин В.А., Кириллов Г.А., Кочемасов Г.Г, Кротов В.А., Кузьмичев Ю.П., Лапин С.Г., Львов Л.В., Мочалов М.Р., Муругов В.М., Осин В.А., Панкратов В.И., Пегоев И.Н., Пунин В.Т., Рядов А.В., Сеник А.В., Соболев С.К., Худиков Н.М., Хрусталев В.А., Чеботарь В.С., Черкесов Н.А., Шемякин В.И. // Квантовая электроника. 1991. Т. 18. № 5. C. 536-537.

[10] Sukharev S.A. // Proc. SPIE. 1999. V. 3492. P. 12-24. 
[11] Ekdahl C.A., Jr. // Beam dynamics for the scorpius conceptual design report. 2017. P. 1-53

[12] Henestroza E., Houck T., Kwan J.W., Leitner M., Miram G., Prichard B., Roy P.K., Waldron W., Westenskow G., Yu S., Bieniosek F.M. // DARHT 2 kA cathode development. 2009. P. 1-115.

[13] Винокуров Н.А., Шевченко О.А. // УФН. 2018. Т. 188. № 5. C. $493-507$. 